\title{
The roles of polarity and symmetry in the perceptual grouping of contour fragments
}

\author{
DAVID J. FIELD ${ }^{1}$, ANTHONY HAYES ${ }^{2, *}$ and ROBERT F. HESS ${ }^{3}$ \\ ${ }^{1}$ Department of Psychology, Cornell University, Ithaca, New York 14853, USA \\ ${ }^{2}$ Department of Psychology, University of Melbourne, Parkville, Victoria 3052, Australia \\ ${ }^{3}$ Department of Ophthalmology, McGill University, Montréal, Québec H3A 1A1, Canada
}

Received 7 December 1998; revised 27 June 1999; accepted 1 September 1999

\begin{abstract}
We describe two experiments that investigate the roles of polarity and symmetry in the perceptual grouping of contour fragments. Observers viewed, for one second on each presentation, arrays of oriented, spatial-frequency band-pass, elements, in which a subset of the elements was aligned along a twisting curve. In each of five conditions we measured observers' ability to detect aligned combinations of even- and odd-symmetric elements, of the same and different polarities, against a background of 'noise' elements. As with previous experiments we found that the 'path' could be reliably detected, even when the elements of the path were oriented at angles of up to $\pm 60 \mathrm{deg}$ relative to each other. Detection of the path was still possible when the polarity of path elements alternated. However, the probability of detection of the path was raised significantly when the path elements were all of the same polarity. Perceptual grouping of even-symmetric elements was no different to perceptual grouping of odd-symmetric elements. The results provide evidence, that in achieving integration of contour fragments, the visual system uses a process that is to some degree phase selective. We use the results to describe how the visual system may resolve natural contours when they occur against backgrounds that vary over a wide range of intensities. The data presented here have been published in conference-abstract form (Hayes et al., 1993; Field et al., 1997).
\end{abstract}

Keywords: Gestalt 'law' of good continuation; phase; visual coding; natural-image contours.

\section{INTRODUCTION}

Much physiological and psychophysical research endorses the idea that neurones in the early visual pathway employ highly localised receptive fields to code the retinal image. The evidence suggests that the retinal image is decomposed at the primary visual cortex by cells selective to local properties of the image such as orientation, spatial frequency, and direction of motion. Local coding of the retinal

\footnotetext{
*To whom correspondence should be addressed. E-mail: a.hayes @ psych.unimelb.edu.au
} 
image by these cells may offer the advantage of the removal of redundancy (see e.g. Field, 1994), but for the purpose of recognition the visual system must unite imagestructure related information carried by a small set of active units; this is sometimes referred to as the 'binding problem'.

One important direction of local unification is along the length of a line or edge, and a number of studies have investigated how the visual system binds the elements of a fragmented contour to result in the perception of unified whole. In earlier work (Field et al., 1993) we established that the visual system groups locally oriented, fragmented, elements of contours by combining the components according to a set of preferences we describe as an 'association field'. As with the 'relatability' notion of Kelman and Shipley (1991), aggregation of locally oriented fragments into visible contours occurs when the fragments align closely to the tangent of smooth curves. Several studies have shown that the grouping of fragments into detectable contours also depends on disparity (Hess and Field, 1995; Hess et al., 1997), hue (Mcllhagga and Mullen, 1996), eccentricity (Hess and Dakin, 1997), number of elements (Moulden, 1994), and closure (Kóvacs and Julesz, 1993; it should be noted that recent evidence suggests that sensitivity to closure may be explained by smoothness constraints on contour integration, see Pettet et al., 1998).

In our earlier work (Field et al., 1993) we investigated the element-alignment rules for the detection of contours where contours were constructed from small separate elements that were set against a background of similar 'noise' elements. Using a two-alternative, forced-choice procedure, observers were required to detect which of two images contained the set of aligned elements or 'path'. Each image consisted of an array of 256, oriented, band-pass 'Gabor-patch' elements (circular, Gaussianwindowed sinusoids), where one image contained a path that consisted of a subset of 12 elements that were aligned along a twisting curve. Observers were capable of detecting a path when the elements of the path differed in their inter-element orientation by up to $60 \mathrm{deg}$. Orienting the elements side-to-side, as opposed to endto-end, decreased substantially the ability of observers to detect the path, as did the introduction of small displacements - either as small lateral displacements or as random orientation jitter - of elements off the axis of the path (see also Beck et al., 1989; Hayes, 1997; Hess et al., 1997). One of the five experiments that were reported in our 1993 study explored the role of the phase of the sinusoid of the Gabor-patch in perceptual grouping of contour fragments. We set the windowed sinusoids of the path elements and of the background elements to random phase, instead of fixed cosine phase (i.e. even symmetry), but the ability of observers to detect the path was unchanged.

This earlier experiment is open to criticism along two lines. First, the elements of the path were relatively narrow-band and thus each element contained several bright and dark bars so, although grouping of the elements may be phase sensitive (e.g. bright-bar to bright-bar and dark-bar to dark-bar), the use of narrow-band stimuli may have precluded measurement of phase selectivity. Second, the phases of the sinusoids of the elements were set to random values. This procedure results in an 
average difference between neighbouring elements of $90 \mathrm{deg}$, and leaves open the possibility that by the more powerful test of alternating the phases of the elements in the path by $180 \mathrm{deg}$ it will be possible to measure phase selectivity. The experiments reported in the present paper were designed to examine this possibility.

\subsection{Defining phase selectivity}

Fourier terminology furnishes a useful addition to the language of visual science (e.g. Campbell and Robson, 1968). Confusion occurs, though, with the use of Fourier terms to describe the properties of cells and/or mechanisms that perform a local analysis. The Fourier 'phase' term is one that has been particularly problematic.

Consider the issue of 'phase selectivity' of cortical simple and complex cells. It is sometimes argued that simple cells are relatively phase sensitive while complex cells are phase insensitive. However, as will be argued below, a cell which shows localisation in the visual field, such as that shown by complex cells, must have a considerable degree of phase sensitivity.

Although simple cells show various non-linearities, their quasi-linear properties result in a high level of phase specificity. Presented with sinusoidal gratings, the cells show strong modulation of their activity at different phases of the sinusoid (Movshon et al., 1978). These cells have been modelled by an envelope and a periodic carrier (e.g. Field and Tolhurst, 1986) where the phase of the carrier relative to the centre of the envelope describes the phase selectivity. Often, phase selectivity is described as the symmetry of the cell where even and odd symmetric functions are defined as follows:

$$
\begin{aligned}
\text { Even symmetric } & \rightarrow f(x)=f(-x) \\
\text { Odd symmetric } & \rightarrow f(x)=-f(-x) .
\end{aligned}
$$

Figure 1 shows examples of even- and odd-symmetric functions that are created by changing the phase of the 'carrier'.



Figure 1. Even- and odd-symmetric functions. The top function is an even-symmetric 'log-Gabor function,' and the middle function is an odd-symmetric 'log-Gabor function.' The bottom function shows the shape of the envelope of either the odd- or even-symmetric function. Unlike a 'Gabor function' (Gaussian-windowed sinusoid), 'log-Gabor functions' (Gaussian amplitude on a log axis in the frequency domain; see Fig. 2d) have the same frequency bandwidth regardless of symmetry (i.e. phase of the 'carrier'). 
It has been proposed that the visual system contains cortical cells that are mostly limited to only even- and odd-symmetric receptive fields (e.g. Pollen and Ronner, 1981; Field and Nachmias, 1984), but the physiological evidence generally supports the notion that cells exist at all modulation phases (e.g. Field and Tolhurst, 1986). Indeed, the receptive fields of many simple cells would appear to be space-time inseparable with, for some forms of inseparability, the modulation phase smoothly progressing as a function of time (see for review, DeAngelis et al., 1995). Despite the lack of evidence for the grouping of cells into even and odd symmetry, Pollen and Ronner (1981) reported that neighbouring cortical cells often differ in phase by $90 \mathrm{deg}$ (i.e. are in quadrature). This pairing would allow the relative phase of a stimulus, falling within the envelope of the overlapping receptive fields of the two cells, to be completely determined in a way similar to how a cosine and a sine coefficient determine the phase of any sinusoid.

Complex cells have sometimes been described as 'phase insensitive' because, when presented with sinusoidal gratings, the response magnitude is often independent of the phase of the sinusoid (e.g. Movshon et al., 1978). However, changing the relative phases of its Fourier components changes the relative position of a stimulus (see Fig. 2). Thus, any cell that has a localised receptive field is necessarily phase selective. A simple cell will respond only when the phases are aligned near the centre of the receptive field AND when the phase of that alignment is appropriate (e.g. in cosine phase for even-symmetric receptive fields). By comparison, complex cells appear to be relatively insensitive to any particular phase of the alignment, though they still require that some alignment occurs in the area of the visual field that is occupied by the receptive field.

\subsection{Phase, and contour integration}

As mentioned above, in our earlier study (Field et al., 1993) we found that the phase value of the elements was not important for contour-element binding. If the phase value plays no role in whether elements group perceptually, then one may be led to conclude that a model employing complex-cell properties would provide a good basis for the integration of the oriented components of the contour.

Caelli et al. (1986) have published demonstrations of texture-element grouping by phase. When even-symmetric elements are surrounded by even-symmetric elements of opposite polarity, the central group of elements readily 'pops out' (i.e. is easily visible). However, when odd-symmetric elements are surrounded by oddsymmetric elements of opposite polarity, the central group does not pop out. This finding suggests that, at least for textures, grouping is phase dependent, but only for even-symmetric elements.

There are several ecological reasons why we may expect contour integration to be phase insensitive. Consider the image shown in Fig. 3a; a picture of a leaf whose edge is sometimes brighter and sometimes darker than the background. Figure $3 b$ shows a bandpass version of the leaf-edge demonstrating how the contrast at the border flips back and forth. In general, any occluding contour in the natural visual 
a
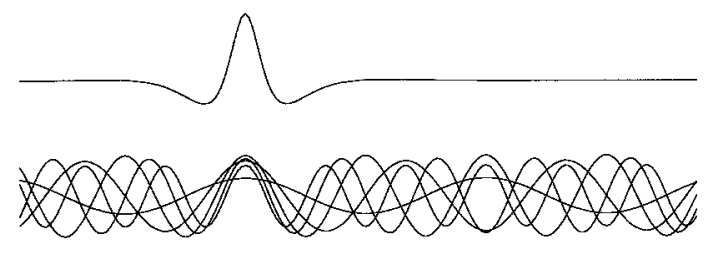

b
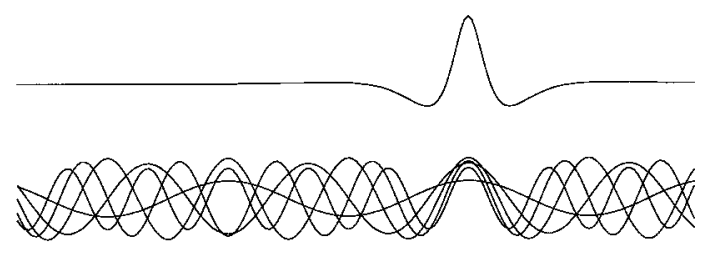

C
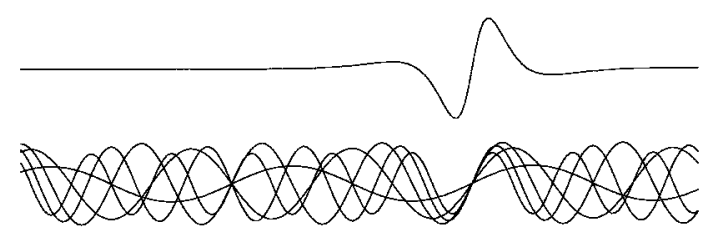

d

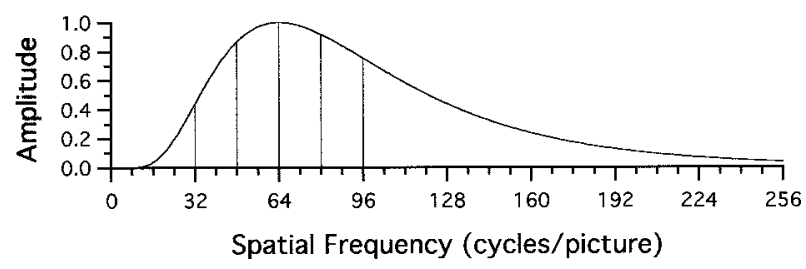

Figure 2. 'Log-Gabor' functions. Beneath each function five spatial-frequency harmonics are shown (scaled, for clarity, but not normalized). Two harmonics fall above the modulation frequency of the log-Gabor function ( 64 cycles/picture), and two harmonics fall below the modulation frequency. The positions on the amplitude spectrum of the log-Gabor function of the depicted harmonics are shown in panel (d). When an even-symmetric function (a) is shifted to the left (b), or converted to an oddsymmetric function (c), no change to the amplitude spectrum of the Fourier transform occurs; these three functions differ only in the phases of their harmonics.

environment will flip back and forth in contrast if it extends across a region of variable luminance. Thus, we might expect an integration process that works effectively with contours in the natural visual environment to be able to integrate contour fragments of opposite polarity. On the other hand, where there are multiple contours, with intersection and overlap as could occur in a cluttered natural scene, the phase of contour fragments may assist in binding those fragments that belong 



Figure 3. Photograph of a leaf (a), and a high-frequency bandpass-filtered image (b). The leaf rests against a background that varies in brightness. The result of this variation is that the contrast of the edge of the leaf changes in magnitude and sign. The flipping of sign is easily seen in the highfrequency bandpass-filtered image (b). Contrast along the leaf edge has been amplified for clarity.

together. In the experiments described below, the role of phase and polarity in contour integration are explored.

\section{METHOD}

The methods and stimuli used in the experiments are the same as used in Field et al. (1993), with two exceptions. First, the individual elements making up the stimulus array had a different spatial profile. Second, the path-construction algorithm prevented the path from wrapping around on itself. These differences are discussed below.

\subsection{Viewing conditions and experimental design}

All experiments used a temporal, two-alternative, forced-choice design. In each experiment a trial consisted of the successive presentation of two images. Each image consisted of an array of 256 elements arranged on a $16 \times 16$ grid; one image contained both background elements and path elements, the other contained only background elements. The order of presentation was randomised from trial to trial.

Each image-presentation was for one second (temporal Gaussian contrast-ramp, one second at half-height) with one second between the two presentations. The path, when it appeared, was constrained to pass through the centre of the array. Observers were required to attend centrally. One second presentation times were chosen since for this duration serial search of the 256 elements of the array, for element pairings that indicate the presence of a path, is not a possible effective 
strategy. With a one-second presentation, though, there is time to make several eye movements. In practise eye movements help little in the task of path detection, and are probably counter-productive, since the only region of the array the path is forced to pass through is the central region. However, the same result, though noisier, can be demonstrated with presentation times of $250 \mathrm{~ms}$ (Field et al., 1993).

The screen was set to the mean grey level between trials. Observers were asked to determine which of a pair of presentations contained a path, indicating their choice of interval by using the left and right buttons of the computer's three-button mouse. This procedure - a 2AFC bias-free technique - ensures the validity of results from observers who are not naive to the aims of the study.

Observers viewed the screen from a distance of $90 \mathrm{~cm}$ under conditions of low ambient illumination. At this distance, each image subtended $8.0 \mathrm{deg}$ of visual angle. The mean luminance of the screen was fixed at $35 \mathrm{~cd} / \mathrm{m}^{2}$. The images were displayed at a resolution of $512 \times 512$ pixels on a Sony Trinitron RGB monitor driven by a Sun SPARC computer. A calibrated lookup table was used to linearize luminance levels, resulting in a display of approximately 7.5 bits (182 grey levels).

Images for all conditions were created before each observation session and stored on the computer's hard disk. The image-pairs consisted of one array of elements that contained no path, and a second array that contained a path. The 'jaggedness' of the path was manipulated by varying the angular difference between path elements. Each block of trials consisted of five interleaved conditions (five different interelement path angles) with 50 trials in each condition. Thus the total number of presentations in any block was 250 . Each block was run four times resulting in a total of 200 trials in each condition. The blocks from each of the five experiments were interleaved, and data collection was conducted over a period of four weeks.

\subsection{Stimulus}

The twisting curve on which the path elements were placed was created using rules described elsewhere (Field et al., 1993). These rules were formulated to ensure that no information about the existence of the path was available to observers, other than the relative orientation of the elements that defined the path. For example, if the spacing of the elements in the path was not equated with the spacing of the background elements, then it would be possible to identify the path simply on the basis of the relative density of elements around the path (see e.g. Uttal, 1975). Control trials were run using circular Gaussian blobs to ensure that artefactual cues, such as spacing or density inhomogeneity, were not available to observers.

For each trial a different example of the path stimulus was generated. Thus the shape of the path and its location (other than that it passed through the central region of the array) was unknown on each presentation. Five examples of the path stimulus, all with a 30 deg inter-element angle (that controlled the amount of twist to the path), are shown in Fig. 4.

In our earlier study the array elements consisted of Gaussian-windowed sinusoids (i.e. Gabor functions). In the current study we needed the elements to be broader in 

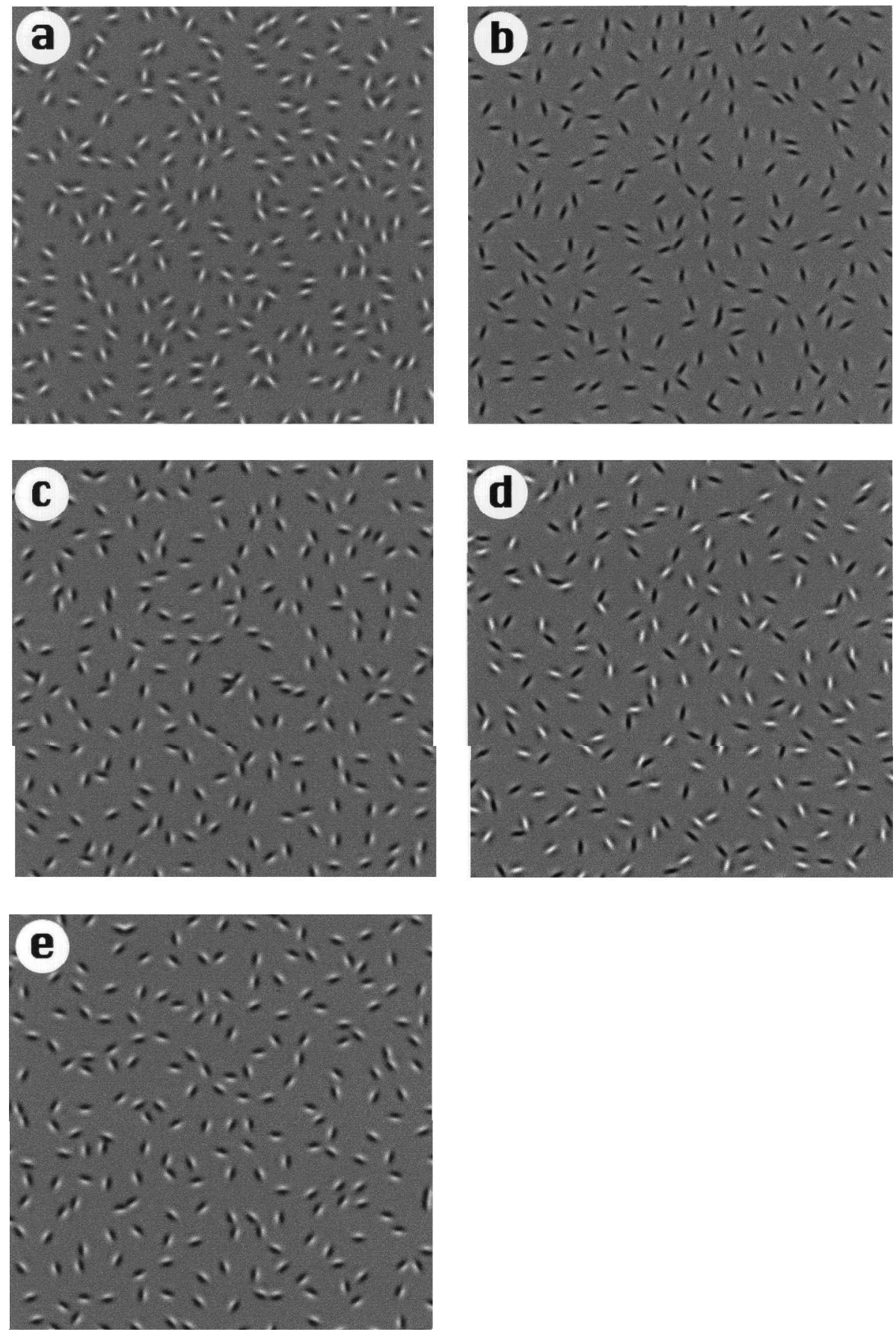

Figure 4. Examples from the five stimulus conditions used in the experiments: (a) even symmetric, bright centre; (b) even symmetric, dark centre; (c) odd symmetric, same polarity; (d) even symmetric, alternating polarity; (e) odd symmetric, alternating polarity. The inter-element angle for the 12element 'paths' shown in each panel is $30 \mathrm{deg} \pm 5^{\circ}$. 
bandwidth and we wanted there to be no variation in bandwidth between even- and odd-symmetric elements. Even-symmetric and odd-symmetric Gabor functions, with identical Gaussian modulation and same frequency of sinusoid, do not have identical amplitude spectra, and this difference is exaggerated as bandwith is increased.

In order that the amplitude spectra of our even- and odd-symmetric functions were the same, we chose to use a similar function to a Gabor function, but one which always has a spectrum which approaches zero amplitude at zero frequency (i.e. the DC equals zero). The function has the following Fourier spectrum:

$$
G(f)=\exp \left\{-\left[\log \left(f / f_{\mathrm{o}}\right)\right]^{2} / 2\left[\log \left(\sigma / f_{\mathrm{o}}\right)\right]^{2} .\right.
$$

This function is referred to as a 'log-Gabor' function, since it has a Gaussian amplitude spectrum when plotted on a log frequency axis. Examples of this function, and the envelope of the function, are shown in Fig. 1. The Fourier amplitude spectrum, on a linear spatial-frequency axis, is shown in Fig. 2d.

For all the experiments the period of modulation was equal to 8.0 pixels, and the width of the elements (full width at half-height) was 8.0 pixels. In all experiments the observers viewed the screen from a distance of $90 \mathrm{~cm}$. These parameters resulted in elements with a peak spatial frequency of 7.6 cycles/degree and a bandwidth of approximately 2.0 octaves. The contrast of each of the elements was the maximum allowed by the screen (95\%). Since the elements did not have significant overlap, the contrast of the entire $512 \times 512$ image was the same as that of the individual elements.

In Field, Hayes and Hess (1993) the path shape was unconstrained in that paths were allowed to loop back onto - though not across - themselves. In the current set of experiments we prevented this possibility, since we had noticed that for images in which paths formed closed loops the paths seemed to show a more vivid pop-out relative to those that did not form closed loops. This effect is beyond the scope of this study and has been the subject of recent work (i.e. Kóvacs and Julesz, 1993; Pettet, McKee, and Grzywacz, 1998).

\subsection{Observers}

Two of the authors, A.H. and D.J.F., served as observers in all experiments. Both were corrected myopes in their late 30's. Both observers were given sufficient practice (typically 100 trials) to reach an asymptote in their performance for each experiment.

\section{EXPERIMENT 1: PATHS OF ODD VERSUS PATHS OF EVEN SYMMETRY}

The first experiment investigated the role of the symmetry of elements for perceptual grouping. In this experiment, all path elements and background elements had the same phase. An example of one of the stimuli is shown in Fig. 4a. In this example, 
all the elements have a bright-bar centre $(P=0 \mathrm{deg})$. Elements of $P=180 \mathrm{deg}$ (dark bar, Fig. 4b), and $P=90 \mathrm{deg}$ (odd-symmetric, Fig. 4c) were also used. The $P=90 \mathrm{deg}$ and the $P=270 \mathrm{deg}$ conditions are equivalent. In all conditions, the background elements had the same phase(s) as the path elements.

\subsection{Results}

The results for the three conditions — even symmetry dark, even symmetry light, and odd symmetry - as a function of the path angle are presented in Fig. 5. The results are in agreement with Field et al., (1993), and show that with an increasing angle of inter-element articulation the detection of the path becomes more difficult. However, observers are still above chance levels of detection of the path even when the inter-element angle is as large as $\pm 60 \mathrm{deg}$. There is no evidence, though, that the observers have different sensitivity to the different stimulus types; in particular there is no difference in performance between the condition where the stimuli are odd-symmetric, and the condition where the stimuli are even-symmetric. In all three conditions the results are similar for both observers.


$\square$ even symmetry, bright centre, same polarity
a even symmetry, dark centre, same polarity

- odd symmetry, same polarity



Figure 5. Results of Experiment 1. Path-detection performance (two alternative, forced choice) measured as a function of path inter-element angle for observers AH and DJF, comparing samepolarity, odd- and even-symmetric, stimulus conditions. Each datum represents the average of four sets of trials; each trial consisted of 50 stimulus presentations. The bar attached to each datum shows \pm 1 SE. The lines show the best-fit weighted error-function to each set of data. The square symbols show results for the even-symmetric stimulus conditions; the round symbols show results for the oddsymmetric stimulus condition. 


\section{EXPERIMENT 2: PATHS CONTAINING ELEMENTS OF ODD AND EVEN SYMMETRY}

In the second experiment the phase of the elements alternated by $180 \mathrm{deg}$. Two conditions were used $-90^{\circ} / 270^{\circ}$ alternation and $0^{\circ} / 180^{\circ}$ alternation. Examples are shown in Figs 4d and 4e.

\subsection{Results}

The results for the two observers are presented in Fig. 6. In Fig. $6 a$ the $0^{\circ} / 180^{\circ}$ results are compared with the 0 deg and 180 deg results of Experiment 1. In Fig. 6b the $90^{\circ} / 270^{\circ}$ results are compared with the 90 deg results of Experiment 1 . In both cases, and for both observers, significantly lower sensitivities were found for the paths with alternating-phase elements.

Figure 6c compares the results from both of the alternating-phase conditions the $0^{\circ} / 180^{\circ}$ condition with the $90^{\circ} / 270^{\circ}$ condition. As with Experiment 1 , there is no significant difference in sensitivity that is dependent on the type of symmetry of the elements.

For both experiments the results of the two observers were similar, and the relevant patterns of the results were identical. Observer D.J.F. tended to be more sensitive than observer A.H. to the presence of a path under all conditions of path inter-element angle, element symmetry, and element polarity.

\section{DISCUSSION}

The results for both observers allow us to draw several conclusions about the role of phase in a contour-integration task under conditions of brief - i.e. $1 \mathrm{~s}-$ presentations of the stimuli. The results suggest that the observers are equally sensitive to paths generated with elements of any phase value, regardless of the value, where the phase value of all elements is the same. Thus, the texturesegregation demonstrations of Rentschler et al. (1988) and Caelli et al. (1986), where odd-symmetric textures do not segregate from a background where the texture and background are of opposite polarities, yet even-symmetric textures will segregate under the same conditions, should not be taken as evidence that either odd- or even-symmetric elements will not group when the task is to find a path. Our results suggest no significant difference for how odd-symmetric and evensymmetric elements bind together.

Texture segmentation can be achieved via two mechanisms - binding within the texture region, and contrast with the background. For our stimuli, binding is the only possible mechanism, since the backgrounds to the path stimuli are in all cases deliberately constrained to have the same composition as the path. We propose that it is likely that the differences in texture segmentation for textures of even and odd symmetry, reported by Rentschler, Hübner and Caelli, result from the differing effectiveness of texture boundary contrasts where texture and background 
a

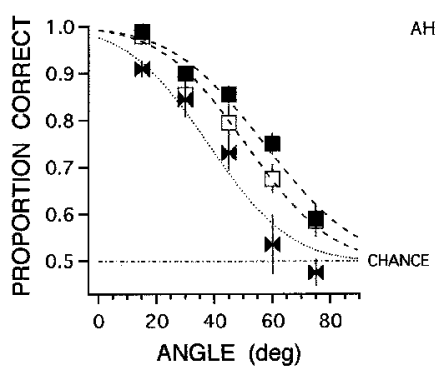

$\mathrm{AH}$

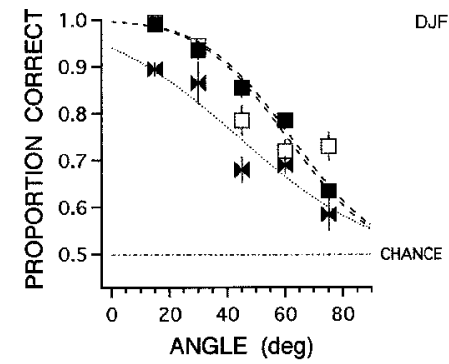

$\square$ even symmetry, bright centre, same polarity

- even symmetry, dark centre, same polarity

* even symmetry, altemating polarity



b
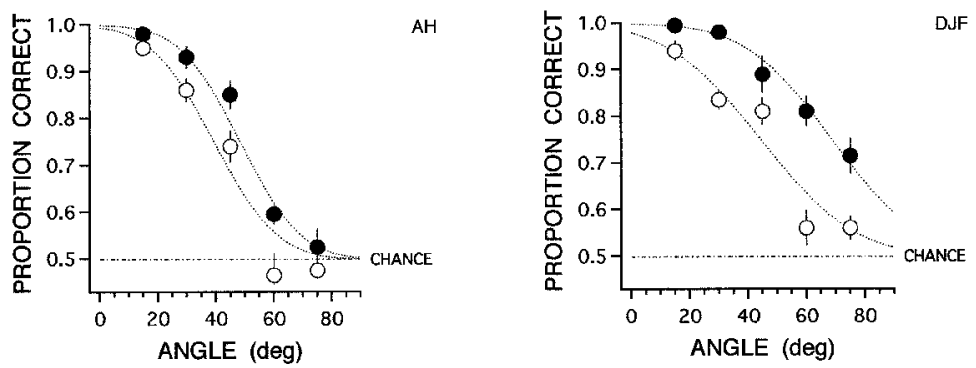

- odd symmetry, same polarity

odd symmetry, alternating polarity

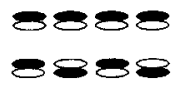

C

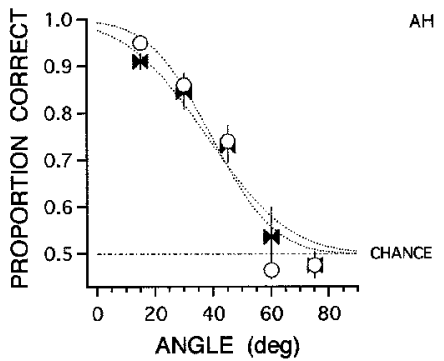

* even symmetry, alternating polarity

odd symmetry, alternating polarity


Figure 6. Results of Experiment 2. Details as for Fig. 5. Panel (a): path-detection performance comparison between even-symmetric, same-polarity (open and closed square symbols), and evensymmetric, alternating-polarity (bow-tie symbols), stimulus condition. Panel (b): path-detection performance comparison between odd-symmetric, same-polarity (closed symbols), and odd-symmetric, alternating-polarity (open symbols), stimulus conditions. Panel (c): path-detection performance comparison between even-symmetric, alternating-polarity (bow-tie symbols), and odd-symmetric, alternating-polarity (round symbols), stimulus conditions. 
are composed of different polarity elements. This proposal is supported by the finding that in general binding within the texture region is less potent for texture segregation than contrast with the background (see Nothdurft, 1994).

Alternating the phases by 180 deg along the path results in a significant reduction in ability to detect the path. The reduction occurs for both even-symmetric and odd-symmetric alternation. This result is important for models of vision that use the envelope, or 'local energy', of the local features because such models, if implemented directly, will fail to predict phase-sensitive results (but see Morrone and Burr, 1988). While the observers were significantly less sensitive to the alternating-phase path, the observers were still significantly above chance. Thus, a phase-insensitive process may play a role in contour-integration.

Taken together, the results suggest that there is both a phase-selective and a non phase-selective component of the process involved in contour integration. There are several physiologically plausible ways this result may be achieved — with the use
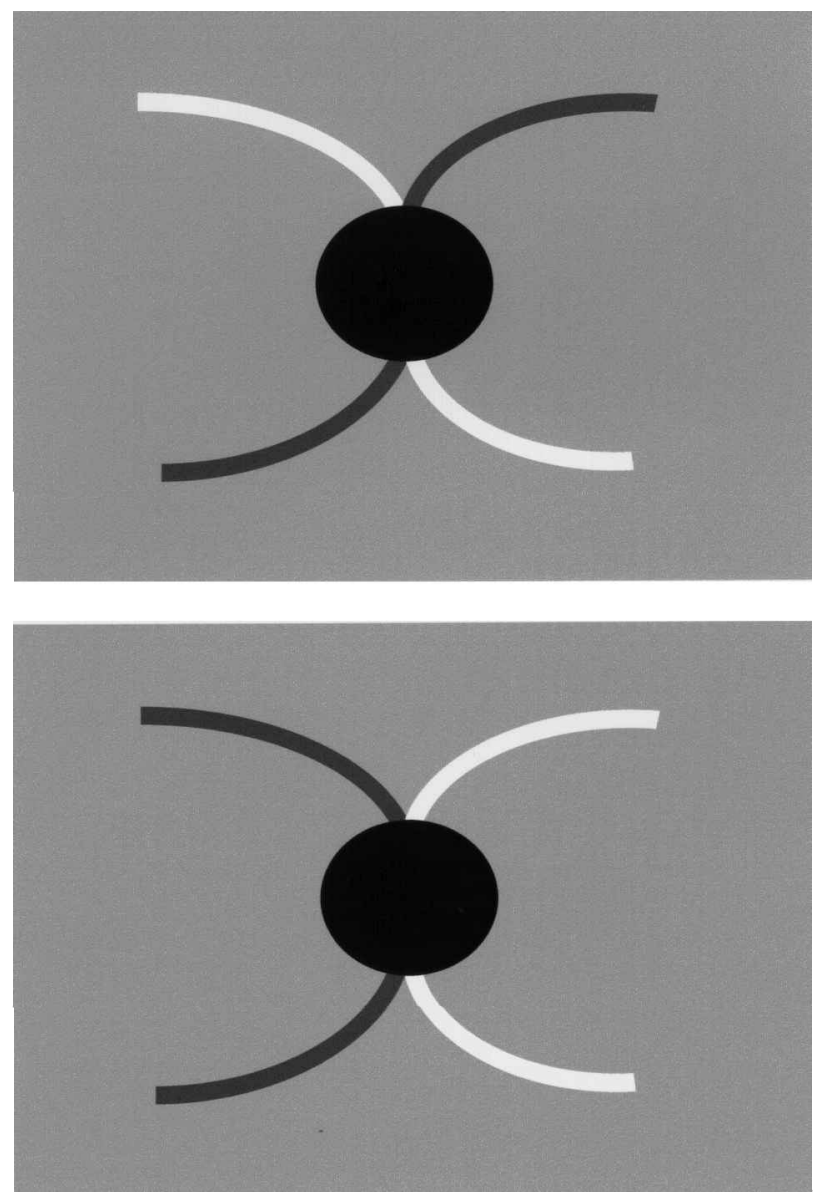

Figure 7. A synthetic image where continuity is resolved by contrast sign. 


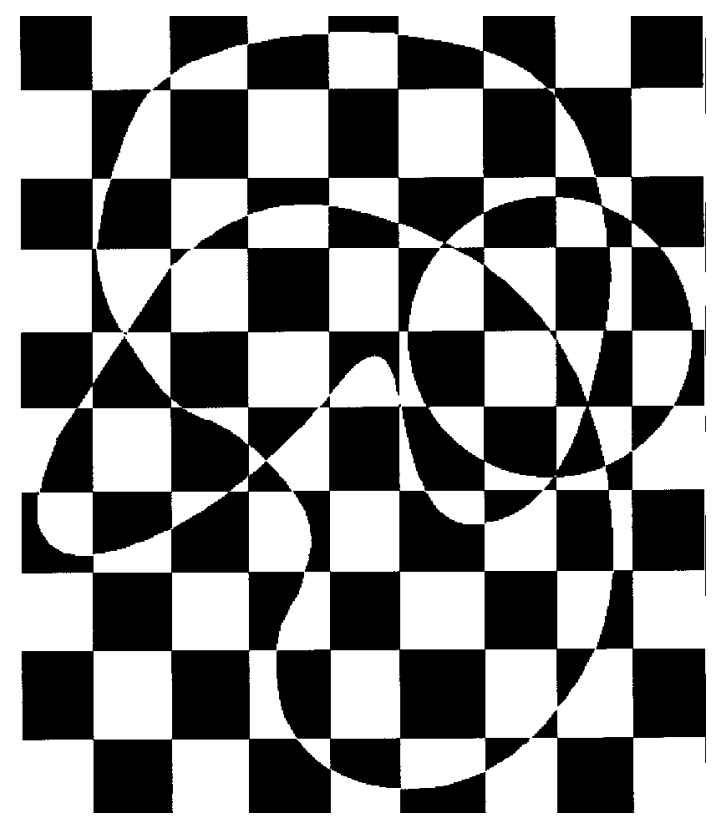

Figure 8. A synthetic image where contour sign flips frequently, but the contour is nevertheless readily perceived.

of phase selective (see also Rentschler, 1985) and phase non-selective mechanisms (e.g. with the use of both simple and complex cells), or as a consequence of the output of a single process that is broadly tuned to phase (Movshon et al. (1978) reported cells in V1 that show a range of phase selectivities).

The finding of Williams and Hess (1998), that inter-element phase differences eliminate flank-element induced, contrast-threshold facilitation (reported by Polat and Sagi, 1993, 1994), coupled with our results, lend weight to speculation (e.g. Kóvacs and Julesz, 1993) that the mechanism responsible for contrast-threshold facilitation, may also be the phase-selective component responsible for contourelement binding.

A phase-insensitive process would be useful for detection of contours such as those shown in Fig. 3, and shown in exaggerated form in Fig. 8. In these images, despite the fact that polarity flips back and forth, the contours are relatively easy to isolate. By contrast, a phase-selective process may help disambiguate situations where edges or lines of different polarities are present in a single image. Consider the image shown in Fig. 7. It is possible to continue the contours as C-shaped curves of two polarities, or vice-versa, or S-shaped curves of a single polarity, or viceversa. The results of Experiment 2 suggest that perceptual grouping is preferred with contour-elements of the same polarity. These results may be used to provide an account for the results of O'Shea and Mitchell (1990), who measured vernier acuity using same-contrast and opposite-contrast stimuli, and found that acuity was higher for same-contrast stimuli. 
Another point should be made about the role of phase-selective contour-element binding in visual processing. As noted in the introduction, one would expect contour integration to be phase insensitive since, for example, occluding boundaries are likely to vary in polarity along the border. However, under some conditions is it important to preserve phase information. Cavanagh and Leclerc (1989) discussed a good example where polarity of a shadow border provides important information about the direction of the shadow, and thus the shape of a 3D object or surface.

Clearly, it can be important that the visual system keep track of the phase, or polarity, of an edge. Also, it is often important to integrate oriented structure along an edge or contour independent of the polarity changes. The work in the present paper suggests that within the visual system processes have developed that are responsive to both of these situations.

\section{Acknowledgements}

We thank two anonymous reviewers for useful comments. This work was supported by a grant from the NIMH to D. J. Field, by a grant from the ARC to A. Hayes, and by a grant from the MRC (Canada) to R. F. Hess.

\section{REFERENCES}

Beck, J., Rosenfeld, A. and Ivry, R. (1989). Line segregation, Spatial Vision 4, 75-101.

Campbell, F. W. and Robson, J. G. (1968). Application of Fourier analysis to the visibility of gratings, J. Physiology 197, 551-566.

Caelli, T., Hübner, M. and Rentschler I. (1986). On the discrimination of micropatterns and textures, Human Neurobiology 5, 129-136.

Cavanagh, P. and Leclerc, Y. G. (1989). Shape from shadows, J. Exp. Psych.: Human Perception and Performance 15, 3-27.

DeAngelis, G. C., Ohzawa, I. and Freeman, R. (1995). Receptive-field dynamics in the central visual pathways, Trends in Neuroscience 18, 451-458.

Field, D. J. (1987). Relations between the statistics of natural images and the response properties of cortical cells, J. Optical Soc. America A 4, 2379-2394.

Field, D. J. (1994). What is the goal of sensory coding? Neural Computation 6, 559-601.

Field, D. J., Hayes, A. and Hess, R. F. (1993). Contour integration by the human visual system: evidence for a local 'association field', Vision Research 33, 173-193.

Field, D. J., Hayes, A. and Hess, R. F. (1997). The role of phase and contrast in contour integration, Investigative Ophthalmology and Visual Science 38, 999.

Field, D. J. and Nachmias, J. (1984). Phase reversal discrimination, Vision Research 24, 333-340.

Field, D. J. and Tolhurst, D. J. (1986). The structure and symmetry of simple-cell receptive-field profiles in the cat's visual cortex, Proc. Royal Soc., London B 228, 379-400.

Hayes, A. (1997). Contour integration with stationary moving elements, Investigative Ophthalmology and Visual Science 38, 74.

Hayes, A., Field, D. J. and Hess, R. F. (1993). Association by the visual system of contour elements of alternating polarity, Perception 22, S5.

Hess, R. F. and Dakin, S. C. (1997). Absence of contour linking in peripheral vision, Nature 390, $602-604$.

Hess, R. F. and Field, D. J. (1995). Contour integration across depth, Vision Research 35, 1699-1711. 
Hess, R. F., Hayes, A. and Kingdom, F. A. A. (1997). Integrating contours within and through depth, Vision Research 37, 691-696.

Hess, R. F., McIlhagga, W. and Field, D. J. (1997). Contour integration in strabismic amblyopia: the sufficiency on an explanation based on positional uncertainty, Vision Research 37, 3145-3162.

Kellman, P. J. and Shipley, T. F. (1991). A theory of visual interpolation in object perception, Cognitive Psychology 23, 141-221.

Kóvacs, I. and Julesz, B. (1993). A closed curve is much more than an incomplete one: Effect of closure in figure-ground segmentation, Proc. Nat. Acad. Sci., USA 90, $7495-7497$.

McIlhagga, W. H. and Mullen, K. T. (1996). Contour integration with colour and luminance contrast, Vision Research 36, 1265-1279.

Morrone, M. C. and Burr, D. C. (1988). Feature detection in human vision: a phase-dependent energy model, Proc. Royal Soc. London B 235, 221-245.

Moulden, B. (1994). Collator units: second-stage orientational filters, in: Symposium on Higherorder Processing in the Visual System, G. R. Bock and J. A. Goode (Eds), Ciba Foundation 184, pp. 170-192. John Wiley, Chichester.

Movshon, J. A., Thompson, I. D. and Tolhurst, D. J. (1978). Spatial summation in the receptive fields of simple cells in the cat's striate cortex, J. Physiology 283, 53-77.

Nothdurft, H.-C. (1994). Common properties of visual segmentation, in: Symposium on Higherorder Processing in the Visual System, G. R. Bock and J. A. Goode (Eds), Ciba Foundation 184, pp. 245-268. John Wiley, Chichester.

O'Shea, R. P. and Mitchell, D. E. (1990). Vernier acuity with opposite-contrast stimuli, Perception 19, $207-221$.

Pettet, M. W., McKee, S. P. and Grzywacz, N. M. (1998). Constraints on long range interactions mediating contour detection, Vision Research 38, 865-879.

Polat, U. and Sagi, D. (1994). The architecture of perceptual spatial interactions, Vision Research 34, $73-78$.

Polat, U. and Sagi, D. (1993). Lateral interactions between spatial channels: Suppression and facilitation revealed by lateral masking experiments, Vision Research 33, 993-999.

Pollen, D. A. and Ronner, S. F. (1981). Phase relationships between adjacent simple cells in the visual cortex, Science 212, 1409-1411.

Rentschler, I. (1985). Symmetry-coded cells in the visual cortex? Nature 317, 581.

Rentschler, I., Hübner, M. and Caelli, T. (1988). On the discrimination of compound gabor signals and textures, Vision Research 28, 279-291.

Uttal, W. (1975). An Autocorrelation Model of Form Detection. Lawrence Erlbaum, Hillsdale.

Williams, C. B. and Hess, R. F. (1998). Relationship between facilitation at threshold and suprathreshold contour integration, J. Opt. Soc. America A 15, 2046-2051. 
Copyright of Spatial Vision is the property of VSP International Science Publishers and its content may not be copied or emailed to multiple sites or posted to a listserv without the copyright holder's express written permission. However, users may print, download, or email articles for individual use. 